University of Nebraska - Lincoln

DigitalCommons@University of Nebraska - Lincoln

$5-1-2000$

\title{
Magnetic properties of self-assembled Co nanowires of varying length and diameter
}

\author{
H. Zeng \\ University of Nebraska - Lincoln
}

M. Zheng

University of Nebraska - Lincoln

Ralph Skomski

University of Nebraska-Lincoln, rskomski2@unl.edu

David J. Sellmyer

University of Nebraska-Lincoln, dsellmyer@unl.edu

Yi Liu

University of Nebraska-Lincoln, yliu@unl.edu

See next page for additional authors

Follow this and additional works at: https://digitalcommons.unl.edu/physicssellmyer

Part of the Physics Commons

Zeng, H.; Zheng, M.; Skomski, Ralph; Sellmyer, David J.; Liu, Yi; Menon, L.; and Bandyopadhyay, Supriyo, "Magnetic properties of self-assembled Co nanowires of varying length and diameter" (2000). David Sellmyer Publications. 65.

https://digitalcommons.unl.edu/physicssellmyer/65

This Article is brought to you for free and open access by the Research Papers in Physics and Astronomy at DigitalCommons@University of Nebraska - Lincoln. It has been accepted for inclusion in David Sellmyer Publications by an authorized administrator of DigitalCommons@University of Nebraska - Lincoln. 
Authors

H. Zeng, M. Zheng, Ralph Skomski, David J. Sellmyer, Yi Liu, L. Menon, and Supriyo Bandyopadhyay 


\title{
Magnetic properties of self-assembled Co nanowires of varying length and diameter
}

\author{
H. Zeng, M. Zheng, R. Skomski, and D. J. Sellmyer \\ Center for Materials Research and Analysis and Department of Physics and Astronomy, \\ University of Nebraska, Lincoln, Nebraska 68588 \\ Y. Liu \\ Center for Materials Research and Analysis and Department of Mechanical Engineering, \\ University of Nebraska, Lincoln, Nebraska 68588 \\ L. Menon and S. Bandyopadhyay \\ Center for Materials Research and Analysis and Department of Electrical Engineering, \\ University of Nebraska, Lincoln, Nebraska 68588
}

Ferromagnetic Co nanowires have been electrodeposited into self-assembled porous anodic alumina arrays. Due to their cylindrical shape, the nanowires exhibit perpendicular anisotropy. The coercivity, remanence ratio, and activation volumes of Co nanowires depend strongly on the length, diameter, and spacing of the nanowires. Both coercivity and thermal activation volume increase with increasing wire length, while for constant center-to-center spacing, coercivity decreases and thermal activation volume increases with increasing wire diameter. The behavior of the nanowires is explained qualitatively in terms of localized magnetization reversal. (C) 2000 American Institute of Physics. [S0021-8979(00)35208-2]

\section{INTRODUCTION}

Ordered nanoarrays have received considerable attention due to the current interest in developing high density magnetic recording media. ${ }^{1}$ Self-assembly is a promising technique for preparing such structures because of its low cost, high yield and the ability to achieve extremely small features. ${ }^{2-5}$ Anodic porous alumina, which has been studied and applied as template material for many years, ${ }^{6}$ is a particularly suitable self-assembled nanochannel material.

When aluminum is anodized in an acid electrolyte, aluminum oxide with nanosized densely packed hexagonal pore array will form. ${ }^{7}$ The diameter, center-to-center spacing between the pores and length of the pores can be easily controlled by varying the electrochemical parameters. Magnetic materials such as $\mathrm{Fe}, \mathrm{Ni}$, and $\mathrm{Co}$ can be grown by electrodeposition as nanowires in such templates. These selfassembled ferromagnetic nanowire arrays are ideal systems to study mesoscopic magnetism, because their radii are comparable to magnetically interesting length scales ${ }^{8}$ of a few $\mathrm{nm}$. In this study, we discuss the dependence of the magnetic properties on the geometry of the Co nanowire arrays.

\section{EXPERIMENT}

The starting material, $99.999 \%$ pure Al foil, was degreased, etched in nitric acid, and electropolished. The foil was then dc anodized in acidic solutions to form a layer of porous alumina. In this study, all the samples were anodized in sulfuric acid under a constant voltage of $10 \mathrm{~V}$, using a platinum cathode. The pores of the anodic alumina were widened while keeping the center-to-center distance fixed by immersing the as-anodized template in phosphoric acid. The ac electrodeposition was carried out at $20 \mathrm{~V}, 150 \mathrm{~Hz}$, using an electrolyte containing $0.1 \mathrm{M} \mathrm{CoSO}_{4}$ and $0.1 \mathrm{M}$ boric acid.

The structure of the deposited material was studied by transmission electron microscope (TEM), high resolution TEM, and nanodiffraction. The magnetic properties of the Co nanowires embedded in the anodic aluminum template were measured by an alternating gradient force magnetometer.

\section{RESULTS AND DISCUSSION}

Figure 1(a) shows the TEM image of ordered Co nanowire arrays, with an average pore diameter around $10 \mathrm{~nm}$, average center-to-center distance between pores about 35 $\mathrm{nm}$, and pore density exceeding $10^{11} / \mathrm{cm}^{2}$. The average wire diameter is roughly equal to the average pore diameter, as evidenced by electron microscopic pictures of nanowires freed from the templates. Figure 1(b) shows the HRTEM top view of a single Co nanowire. The polycrystalline nature of the Co nanowire is evident from the lattice fringes in the HRTEM picture. Typically, the plane view of each Co nanowire reveals three to five grains, with average diameter of about $3-5 \mathrm{~nm}$. The orientation of the lattice fringes varies from grain to grain both inside the wire and between wires, and overlapping of the grains is shown by the Moire fringes. The nanodiffraction patterns show that the crystal structure of the Co nanowires is hcp. We observe no preferential ori- 


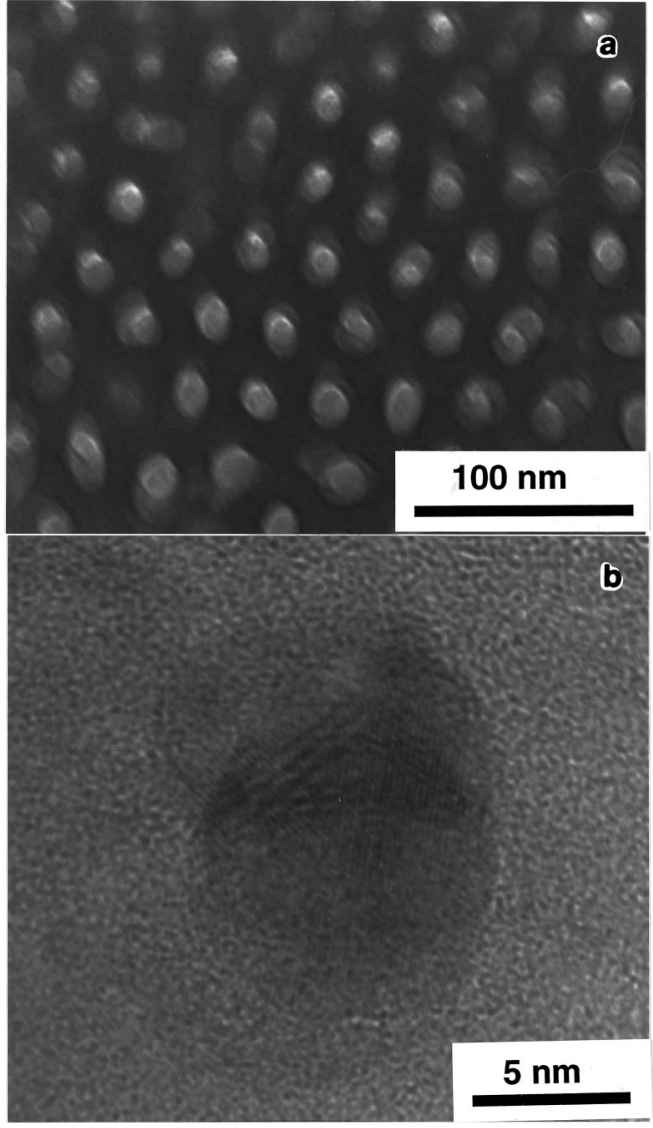

FIG. 1. Top view of acicular Co particles deposited in anodic porous alumina; (a) TEM image and (b) typical HRTEM micrograph. In (b), the sets of parallel lines (lattice fringes) are associated with the orientation of the crystallites.

entation of Co $c$-axis. By contrast, Li and Metzger ${ }^{9}$ found that $\mathrm{Fe}$ particles deposited in anodic alumina are single crystallites.

Typical hysteresis loops for Co nanowires in an anodic alumina template, with aspect ratio greater than 20 , is shown in Fig. 2. The sample possesses uniaxial anisotropy, with the

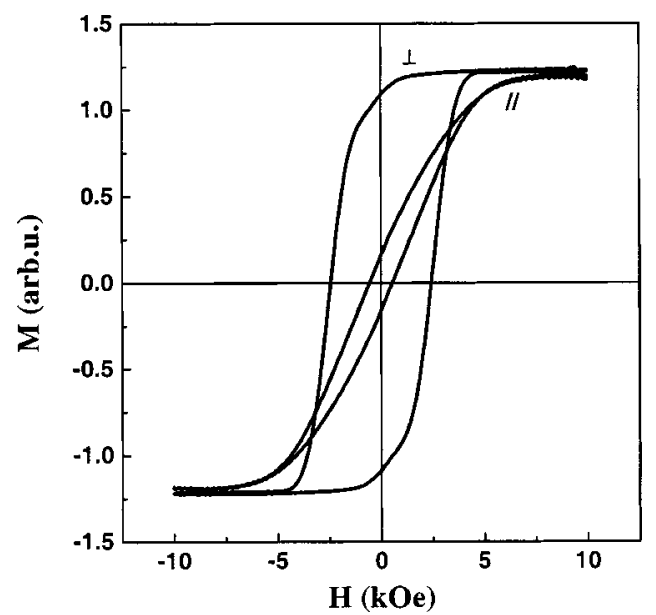

FIG. 2. Hysteresis loops of Co nanowire arrays with a diameter about $10 \mathrm{~nm}$ and center to center distance of about $35 \mathrm{~nm}$ measured parallel and perpendicular with respect to the film plane.
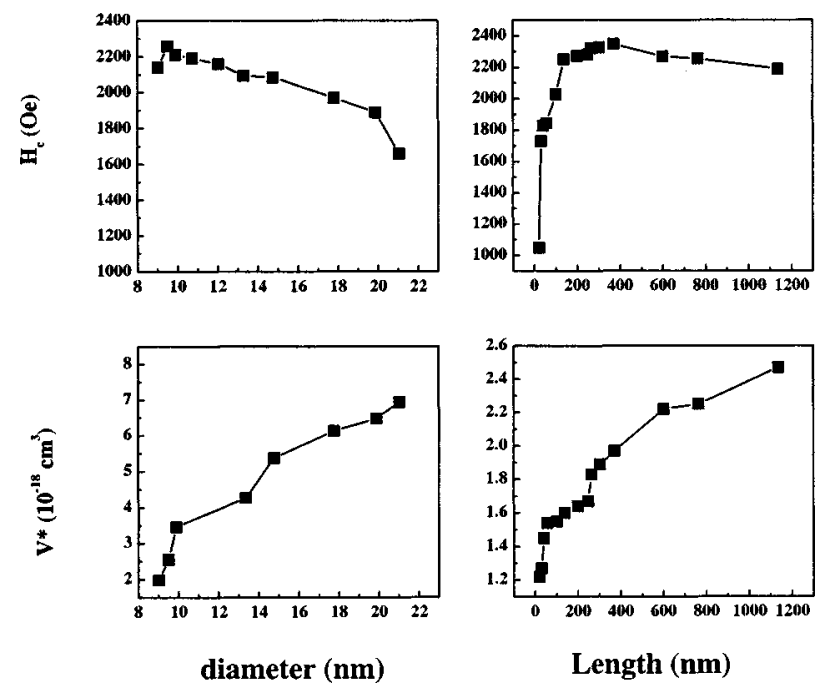

FIG. 3. Coercivity $H_{c}$ and activation volume $V^{*}$ as a function of nanowire diameter (left) and length (right).

easy axis along the wire and perpendicular to the film plane. We expect the uniaxial anisotropy to originate mainly from shape anisotropy, due to the large aspect ratio of the nanowire. Since the TEM results show the polycrystalline nature of the nanowire, with small grains and no preferential orientation of Co $c$-axis, magnetocrystalline anisotropy should play a minor role. The anisotropy field $H_{k}$ is obtained by extrapolating the magnetization curves. $H_{k}$ for samples with an aspect ratio greater than 10 is about $7 \mathrm{kOe}$, which is close to the theoretically predicted shape anisotropy field $2 \pi M_{s}$ (8 kOe for Co) for an infinite cylinder. Perpendicular coercivity as high as $2.6 \mathrm{kOe}$, and remanence ratio exceeding 0.9 are obtained.

As mentioned above, the dimensions of the nanowire arrays can be controlled by varying the electrochemical conditions. For example, the diameter of the wire can be enlarged by immersing the as-anodized template into phosphoric acid for different durations. The average length of the nanowires can be controlled by varying the electrodeposition time. Figure 3 gives the change of coercivity $H_{c}$ and thermal activation volume $\left(V^{*}\right)$ as a function of nanowire diameter $\left(d_{w}\right)$, and length $\left(L_{w}\right)$. For samples with varying length, $d_{w}$ is fixed at $10 \mathrm{~nm}$, while $L_{w}$ changes from $10 \mathrm{~nm}$ to $1000 \mathrm{~nm}$; for samples with varying diameter, the center-to-center distance $d_{c}$ is fixed at $40 \mathrm{~nm}, L_{w}$ is between $500 \mathrm{~nm}$ and 1000 $\mathrm{nm}$, while $d_{w}$ changes from $9 \mathrm{~nm}$ to $20 \mathrm{~nm}$, so that the aspect ratio is kept greater than 25 . The thermal activation volume is used to investigate the energy barriers responsible for the magnetization reversal mechanism. We have determined $V^{*}$ exploiting sweep-rate dependence of coercivity ${ }^{10}$ and magnetic viscosity measurements. ${ }^{11}$ In many cases where the results have been compared, these two methods agree with each other well, so that we will restrict ourselves to the former method.

With an increase of the nanowire length, $H_{c}$ increases steeply until a constant value of about 2300 Oe is approached at $L_{w}$ of about $200 \mathrm{~nm}$. $V^{*}$ first increases rapidly then gradually with $L_{w}$, but is generally much smaller than 


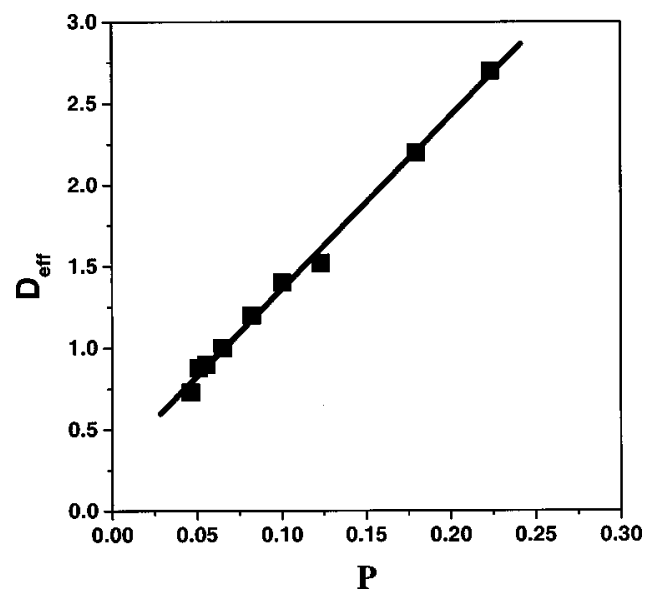

FIG. 4. $D_{\text {eff }}$ as a function of packing density $P$.

the physical wire volumes, which indicates that the reversal starts in a small region of wires. A simultaneous fitting of $H_{c}$ and $V^{*}$ as a function of $L_{w}$ is given in Ref. 15. On the other hand, the coercivity decreases gradually with increasing nanowire diameter accompanying the shearing of the hysteresis loop. Both the decrease of $H_{c}$ and the shearing of the loop can be attributed to magnetostatic interactions. A $\Delta m$ plot $^{12}$ is usually employed to investigate intergranular interactions. For our samples, all the $\Delta m$ peaks are negative, which suggests that the dominant interaction is magnetostatic. However, the interpretation of $\Delta m$ for a material with perpendicular anisotropy is not easy, because of the existence of a macroscopic demagnetizing field. Hence, in order to investigate inter wire interaction, we use an effective demagnetizing factor $D_{\text {eff }}$, as estimated from the skewing of the hysteresis loops, on the assumption that the slope $d M / d H$ at $H_{c}$ is infinite for noninteracting, well-aligned nanowires. For samples with varying $d_{w}$, the demagnetizing field due to individual wires can be neglected since the aspect ratio is large, and $D_{\text {eff }}$ can be attributed totally to mutual interaction between wires. As shown in Fig. 4, $D_{\text {eff }}$ increases with packing density $P$, which is given by $\left(d_{w} / d_{c}\right)^{2} / 2 \sqrt{3}$ for hexagonal arrays. A mean-field theory calculation predicts that $D_{\text {eff }}=4 \pi P .{ }^{13}$ In Fig. 4, a slope of 10.6 is obtained from the linear fitting of $D_{\text {eff }}$ as a function of $P$. Considering the simple assumption we made to estimate $D_{\text {eff }}$ our results agree reasonably with the theoretical prediction. It is generally difficult to interpret $D_{\text {eff }}$ as a function of $L_{w}$, since a proper separation of the demagnetizing field due to individual wires and the interaction between wires is generally nontrival. ${ }^{14}$

Frequently considered magnetic reversal mechanisms such as coherent rotation, and curling treat the wires as perfect homogeneous cylinders. However, the coherent rotation and curling modes are delocalized and could lead to activation volumes much larger than those observed. A more reasonable explanation of the reduced coercivities and thermal activation volumes encountered in practice is morphological inhomogenities. In particular, our TEM results reveal a considerable degree of polycrystallinity with crystallite diameter of about $5 \mathrm{~nm}$. Polycrystalline wires can be interpreted as random-anisotropy ferromagnets where interatomic exchange tries to align the local spins, but the exchange stiffness has to compete against random-anisotropy forces associated with the local uniaxial anisotropy. As a consequence, the magnetization processes become localized. The localization length or physical activation volume strongly depends on the crystallite size. Details of the theoretical explanation, which involves scaling laws and crossover behavior as a function of wire dimensions, will be published elsewhere. ${ }^{15}$

Summarizing our results, we have investigated structural and magnetic properties of Co nanowire arrays electrodeposited into porous anodic alumina. The coercivity, activation volume, and other magnetic properties depend on the length and diameter of the nanowire. Magnetic and thermal activation volume measurements indicate localized reversal, that is, the reversal starts in a region much smaller than the wire volume. This localization is ascribed to the random anisotropy associated with the polycrystallinity of the wires.

\section{ACKNOWLEDGMENTS}

The research is supported by NSF, IBM, CMRA, and ARO.

${ }^{1}$ S. Y. Chou, M. S. Wei, P. R. Krauss, and P. B. Fisher, J. Appl. Phys. 76, 6673 (1994).

${ }^{2}$ D. AlMawlawi, N. Coombs, and M. Moskovits, J. Appl. Phys. 70, 4421 (1991).

${ }^{3}$ X. H. Bao, F. Y. Li, and R. M. Metzger, J. Appl. Phys. 79, 4866 (1996).

${ }^{4}$ G. T. A. Huysmans and J. C. Lodder, J. Appl. Phys. 64, 2016 (1988).

${ }^{5}$ J.-E. Wegrowe, D. Kelly, Y. Jaccard, Ph. Guittienne, and J.-Ph. Ansermet, Europhys. Lett. 45, 626 (1999).

${ }^{6}$ F. Keller, M. S. Hunter, and D. L. Robinson, J. Electrochem. Soc. 100, 411 (1953).

${ }^{7}$ H. Masuda and K. Fukuda, Science 268, 1466 (1995).

${ }^{8}$ R. Skomski and J. M. D. Coey, Permanent Magnetism (Institute of Physics, Bristol, 1999).

${ }^{9}$ F. Y. Li and R. M. Metzger, J. Appl. Phys. 81, 3806 (1997).

${ }^{10}$ P. Bruno, G. Bayreuther, P. Beauvillain, C. Chappert, G. Lugert, D. Renard, J. P. Renard, and J. Seiden, J. Appl. Phys. 68, 5759 (1990).

${ }^{11}$ R. Street and J. C. Woolley, Proc. Phys. Soc., London, Sect. A 62, 562 (1949).

${ }^{12}$ P. E. Kelly, K. O'Grady, P. I. Mayo, and R. W. Chantrell, IEEE Trans. Magn. MAG-25, 3881 (1989).

${ }^{13}$ R. Skomski, H. Zeng, and D. J. Sellmyer (unpublished).

${ }^{14}$ R. Skomski, J. P. Liu, and D. J. Sellmyer, Phys. Rev. B 60, 7359 (1999).

${ }^{15}$ R. Skomski, H. Zeng, M. Zheng, and D. J. Sellmyer, Phys. Rev. B. (submitted). 